\title{
A Novel Self-Microemulsifying System for the Simultaneous Delivery and Enhanced Oral Absorption of Curcumin and Resveratrol
}

Authors

Patcharawalai Jaisamut ${ }^{1,2}$, Kamonthip Wiwattanawongsa ${ }^{1,3}$, Ruedeekorn Wiwattanapatapee ${ }^{1,2}$

\section{Affiliations}

1 Department of Pharmaceutical Technology, Faculty of Pharmaceutical Sciences, Prince of Songkla University, Hat-Yai, Songkhla, Thailand

2 Phytomedicine and Pharmaceutical Biotechnology Excellence Research Center, Faculty of Pharmaceutical Sciences, Prince of Songkla University, Hat-Yai, Songkhla, Thailand

3 Department of Clinical Pharmacy, Faculty of Pharmaceutical Sciences, Prince of Songkla University, Hat-Yai, Songkhla, Thailand

\section{Key words}

self-microemulsifying drug delivery system, curcumin, resveratrol, oral absorption, co-delivery

$\begin{array}{ll}\text { received } & \text { January 27, 2016 } \\ \text { revised } & \text { April 21, 2016 } \\ \text { accepted } & \text { May 2, 2016 }\end{array}$

accepted May 2, 2016

\section{Bibliography}

DOI http://dx.doi.org/10.1055/s-0042-108734

Published online June 9, 2016 | Planta Med 2017; 83: 461-467

(c) Georg Thieme Verlag KG Stuttgart · New York I ISSN 0032-0943

\section{Correspondence}

Ruedeekorn Wiwattanapatapee, PhD

Department of Pharmaceutical Technology, Faculty of Pharmaceutical

Sciences, Prince of Songkla University

15 Karnjanavanich Rd., Hat Yai, Songkhla, 90112, Thailand

Phone: +6674288915 , Fax: +6674428148

ruedeekorn.w@psu.ac.th

\begin{abstract}
The use of curcumin and resveratrol in combination has now become increasingly of interest because of their synergistic effects as therapeutic agents for various diseases, especially cancer. To overcome the poor oral bioavailability of both compounds and improve patient compliance, a novel self-microemulsifying formulation containing curcumin together with resveratrol was developed. Capryol 90, Cremophor $\mathrm{EL}$, and Labrasol were selected as the oil, surfactant, and co-surfactant in the formulation, respectively, based on the solubility study of both compounds. More than $70 \%$ and $80 \%$ of curcumin and resveratrol, respectively, were released in $20 \mathrm{~min}$. The formulation formed a fine oil in water microemulsion with droplet sizes in aqueous media of 15$20 \mathrm{~nm}$. In addition, the formulation containing curcumin and resveratrol showed greater antioxidant activity than that of the formulations with individual compounds, while the cytotoxic activity against HT-29 of the co-formulation $\left(\mathrm{IC}_{50}=18.25 \mu \mathrm{M}\right.$; curcumin and resveratrol in the ratio $1: 1$ ) was less than the formulation with only curcumin $\left(\mathrm{IC}_{50}=30.1 \mu \mathrm{M}\right)$ and only resveratrol $\left(\mathrm{IC}_{50}=25.4 \mu \mathrm{M}\right)$. After oral administration to rabbits, the self-microemulsifying formulation containing curcumin together with resveratrol increased the total plasma concentrations of curcumin and resveratrol by 10 -fold and 6 -fold, respectively, compared to the unformulated combination. This study clearly demonstrated the potential use of the self-microemulsifying formulation for co-delivery, and enhanced oral absorption of poorly watersoluble natural compounds. In addition, the combination was found to produce synergistic antioxidant activity and cytotoxicity against HT-29 cells.
\end{abstract}

\section{Introduction}

Diferuloylmethane, commonly called curcumin, is a yellow pigment present in the rhizomes of turmeric (Curcuma longa L., Zingiberaceae). Curcumin exhibits antioxidative, anti-inflammatory, anticarcinogenic, and chemopreventive properties. In clinical studies, curcumin has been shown to prevent or treat various cancers in humans [1]. Resveratrol (3,5,4'-trihydroxystilbene) is a major component of grapes, wine, peanuts, and Polygonum cuspidatum Sieb. \& Zucc. (Polygonaceae). It also has anticancer, antioxidant, and anti-inflammatory activities [2]. Recently, curcumin and resveratrol, two particularly important polyphenolic compounds, have been found to exhibit a synergistic anticancer effect against various cancer types, including colon cancer and hepato- cellular carcinoma, and also in the treatment of diseases associated with oxidative stress [3].

However, both curcumin and resveratrol have low aqueous solubility and are rapidly metabolized. These problems result in poor oral bioavailability [4], which is an important restriction on their therapeutic usefulness. Many studies have indicated the importance of using self-microemulsifying drug delivery systems (SMEDDS) to improve solubility, absorption, and to increase the oral bioavailability of poorly water-soluble drugs [5]. SMEDDS are defined as isotropic mixtures of oils, surfactants, and co-solvents/ co-surfactants that emulsify under conditions of gentle agitation, similar to those encountered in the gastrointestinal tract [6]. The spontaneous formation of an emulsion presents the drug in a dissolved form, and the resultant small droplet sizes provide a large interfacial surface area that enhances the rate and extent of oral absorption [7]. 
In order to enhance the solubility and oral absorption of both compounds, a new self-microemulsifying formulation containing curcumin in combination with resveratrol (CR-SME) was developed. The synergistic antioxidant activity and cytotoxicity of the new formulation against HT-29 cells were also evaluated. It is expected to be a promising approach to improve the performance of medicines and functional foods used to prevent and treat some important diseases in the future.

\section{Results and Discussion}

Suitable excipients in a self-microemulsifying system should have good solubilizing properties for the drug combination and form a monophasic liquid at an ambient temperature. The solubility in various vehicles of curcumin and resveratrol in combination is presented in - Table 1 . The combination had a higher solubility in Cremophor EL than in other vehicles with $85.98 \pm 0.67$ and $110.84 \pm 0.54 \mathrm{mg} / \mathrm{mL}$ of curcumin and resveratrol, respectively, therefore, Cremophor EL was selected as a surfactant. The result might be due to the ability of the two polyphenols to form hydrogen bonds with the polyethylene oxide (PEO) groups [8]. In the same manner, Labrasol as a co-surfactant, composed of PEO groups, also exhibited a high solubilization capacity for the curcumin/resveratrol combination. Polyethylene glycol (PEG) 400 had a high solubilizing capacity for both compounds. These probably involved hydrophobic interactions between the ethylene units of PEG and the aromatic rings of the compounds [9], but the formulation could not form a translucent microemulsion upon dilution. In the case of Transcutol HP, it was not chosen to be a co-surfactant due to the color change of the produced solution. Among the oily phase compounds, Capryol 90 provided the highest solubility for both curcumin and resveratrol at $6.71 \pm 0.07 \mathrm{mg} / \mathrm{mL}$ and $17.35 \pm 0.02 \mathrm{mg} / \mathrm{mL}$, respectively.

Ternary phase diagrams were constructed to study the proportion of components that can produce the best microemulsion. The mixture of Cremophor EL, Capryol 90, and Labrasol at the ratio $85: 10: 5$ by weight was chosen as the best system for the curcumin/resveratrol combination. This proportion can totally dissolve both compounds and there was no sign of a phase separation into a cloudy emulsion upon dilution with water in different ratios $(1: 10,1: 100$, and $1: 1000)$. The liquid CR-SME formulation of $900 \mathrm{mg}$ (1 capsule) contained $25.5 \mathrm{mg}$ of each curcumin and resveratrol.

Emulsion droplet size plays a vital role in the oral delivery of SME. A uniform and small particle size has an influence on the transport of a drug for delivery to a specific target [10]. The average droplet size of microemulsion from the formulation without compound (blank-SME) and CR-SME after dilution with water was $13.10 \pm 0.40 \mathrm{~nm}$ and $15.90 \pm 0.10 \mathrm{~nm}$, respectively. In this study, deionized (DI) water, simulated gastric fluids (SGF), and simulated intestinal fluids (SIF) were used as a medium for the dispersal of a microemulsion from the CR-SME formulation. The average particle sizes in the DI water, SGF, and SIF were $15.85 \pm 0.07 \mathrm{~nm}, 18.29 \pm 0.04 \mathrm{~nm}$, and $20.15 \pm 0.37 \mathrm{~nm}$, respectively ( $\bullet$ Fig. 1$)$. The polydispersity index (PDI) was in the range of $0.075-0.125$. This result indicated that the types of media had
- Table 1 The solubility of curcumin in combination with resveratrol in various vehicles.

\begin{tabular}{|c|c|c|}
\hline Vehicles & $\begin{array}{l}\text { Curcumin solubility } \\
(\mathrm{mg} / \mathrm{mL}) \\
\text { mean } \pm \text { S.D. }(n=5)\end{array}$ & $\begin{array}{l}\text { Resveratrol solubility } \\
(\mathrm{mg} / \mathrm{mL}) \\
\text { mean } \pm \text { S.D. }(\mathrm{n}=5)\end{array}$ \\
\hline \multicolumn{3}{|l|}{ Oils } \\
\hline Corn oil & $0.39 \pm 0.01$ & $0.11 \pm 0.01$ \\
\hline Capryol 90 & $6.71 \pm 0.07$ & $17.35 \pm 0.02$ \\
\hline Labrafac PG & $0.90 \pm 0.01$ & $0.31 \pm 0.17$ \\
\hline Labrafac CC & $0.78 \pm 0.03$ & $0.23 \pm 0.15$ \\
\hline Soyabean oil & $0.23 \pm 0.02$ & $0.12 \pm 0.11$ \\
\hline \multicolumn{3}{|l|}{ Surfactants } \\
\hline Capryol PGMC & $13.93 \pm 0.07$ & $16.83 \pm 0.05$ \\
\hline Cremophor EL & $85.98 \pm 0.67$ & $110.84 \pm 0.54$ \\
\hline Cremophor RH 40 & $3.11 \pm 0.04$ & $2.46 \pm 0.14$ \\
\hline Labrafil M 2125 CS & $0.60 \pm 0.01$ & $2.38 \pm 0.07$ \\
\hline Lauroglycol 90 & $4.24 \pm 0.09$ & $10.67 \pm 0.02$ \\
\hline \multicolumn{3}{|l|}{ Co-surfactants } \\
\hline Labrasol & $62.99 \pm 0.38$ & $69.25 \pm 0.17$ \\
\hline Lauroglycol FCC & $0.08 \pm 0.01$ & $1.20 \pm 0.08$ \\
\hline PEG 400 & $74.49 \pm 0.20$ & $66.68 \pm 0.15$ \\
\hline Propylene glycol & $12.57 \pm 0.53$ & $18.25 \pm 0.02$ \\
\hline Transcutol HP & $70.13 \pm 0.36$ & $76.50 \pm 0.23$ \\
\hline
\end{tabular}

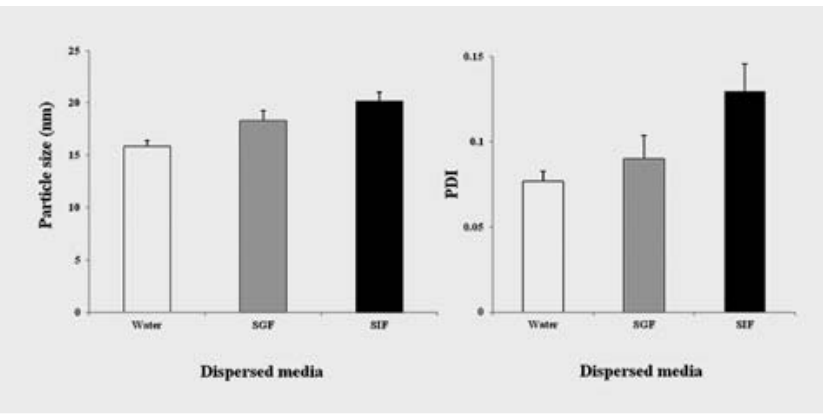

- Fig. 1 The particle size and polydispersity index (PDI) of the CR-SME formulation diluted by different dispersed media $(n=3)$. SGF: simulated gastric fluid; SIF: simulated intestinal fluid.

some influence on the particle size and PDI. Generally, the small oil droplet size was observed in the presence of high amounts of surfactant in the formulation. There is a possibility that Cremophor EL, a nonionic surfactant was able to solubilize a hydrophobic drug ( $\mathrm{HLB}=12-14)$, promoted the small-sized particles and augmented the entrapment property of the combination for delivery [11]. Transmission electron microscopy observations were performed with a volume ratio of the CR-SME/water at $1 / 75$. This demonstrated the spherical shape of the particles with no signs of coalescence, even after $24 \mathrm{~h}$ of dilution. The image is shown in $>$ Fig. 2 in which the average diameter of this formulation was less than $30 \mathrm{~nm}$, which agreed with the data obtained by the dynamic light scattering technique. 
The in vitro release profiles of curcumin and resveratrol from the SME formulation in SGF, pH 1.2, are shown in > Figs. 3 and $\mathbf{4}$. This CR-SME formulation exhibited an immediate release of curcumin, with over $70 \%$ of curcumin released within 20 min, which was similar to that from the self-microemulsifying formulation with individual curcumin (C-SME; - Fig. 3). In contrast, only $5 \%$ of the curcumin was released from the non-formulated combination. A rapid release of resveratrol from the CR-SME formulation was also found with over $80 \%$ within $20 \mathrm{~min}$, and in a similar manner to that from the self-microemulsifying formulation with individual resveratrol (R-SME; $>$ Fig. 4 ), whereas only $18 \%$ of the dose was released from the non-formulated combination. These results demonstrated that the release profile of each polyphenol, either curcumin or resveratrol, was not affected by the presence of the other ingredients in the formulation. It should be noted, however, that resveratrol exhibited a greater percentage release than curcumin, because of its greater number of hydroxyl groups that led to an increased association of interactions with water. The hydrogen bonding of both the polyphenol compounds seemed to be a vital variable during the solubilization process [12]. The stability of the CR-SME formulation was evaluated for its physicochemical properties in the intermediate condition $\left(30 \pm 2{ }^{\circ} \mathrm{C} / 65 \pm 5 \% \mathrm{RH}\right)$ compared to the accelerated stress condition of $45^{\circ} \mathrm{C} / 75 \% \mathrm{RH}$ for 0,1 , and 3 months. A brown glass or container with light protection is recommended for storage of the CR-SME due to the resveratrol undergoing photodegradation, and curcumin is also sensitive when exposed to light [13]. From the results of $\boldsymbol{\nabla}$ Table 2 , the CR-SME formulation showed no significant change in appearance after 3 months of storage in both conditions. The emulsion droplet size was in the range of $19.4-21.5 \mathrm{~nm}$. The liquid formulation was found to be stable, and there was no change in the content of curcumin (99-101\%) and resveratrol (101-102\%).

Among the several types of cells, HT-29 (human colon adenocarcinoma cell lines) is frequently used as an in vitro cancer model. In order to test the cytotoxicity of each compound and the CR-SME formulations against HT-29 cells, MTT assays were carried out. The results in $\boldsymbol{\nabla}$ Fig. $\mathbf{5}$ clearly showed that the CR-SME (the combination ratio for curcumin/resveratrol was $1: 1$ ) had a lower $\mathrm{IC}_{50}$ than each separate polyphenol used in the SME $(18.25 \mu \mathrm{M}$ for CR-SME, 25.4 $\mu \mathrm{M}$ for R-SME, and $30.1 \mu \mathrm{M}$ for C-SME). However, the blank SME had an $\mathrm{IC}_{50}$ value of $100 \mu \mathrm{g} / \mathrm{mL}$. As Cremophor EL was the main component in this formulation, it might further increase the therapeutic effect of some anticancer agents to produce oxidative stress [14]. In a similar previous study from Majumdar et al. [3], they reported that curcumin in combination with resveratrol was a more effective chemopreventive agent than each polyphenol alone. In this study, it has been demonstrated that the co-delivery treatment of polyphenols prepared in the SME formulation caused greater inhibition of colon cancer cells than using each compound in the same system.

To test the combined antioxidant effect of curcumin and resveratrol in the SME, the ferric reducing antioxidant power (FRAP) assay was performed. The CR-SME formulation produced a higher absorbance at $590 \mathrm{~nm}$ than both agents alone in the formulation (C-SME and R-SME) and the blank SME ( Fig. 6). The number and position of the hydroxyl groups in phenolic acids play an important role in their antioxidant activity. The antioxidant activity of

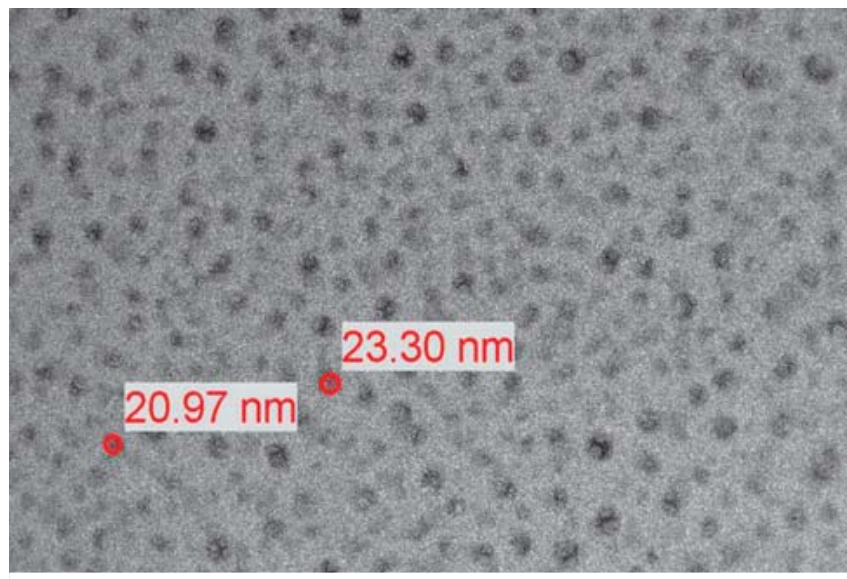

- Fig. 2 TEM micrographs of the CR-SME formulation ( $\times 50000)$. $\mathrm{Bar}=200 \mathrm{~nm}$.

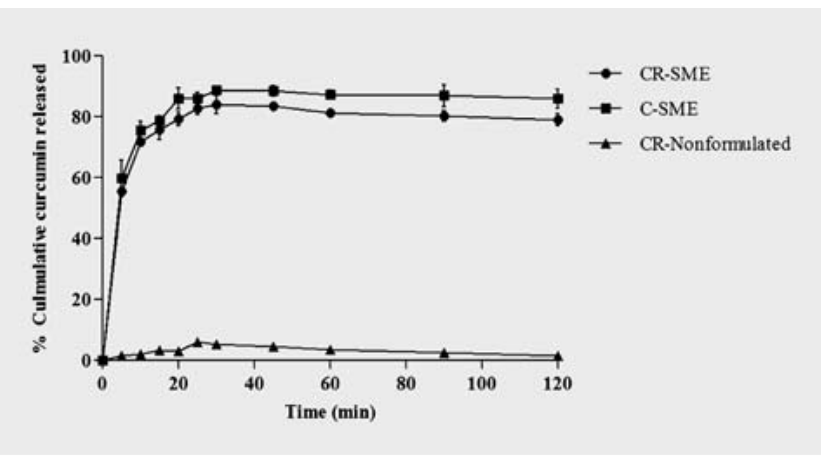

- Fig. 3 The cumulative release of curcumin from a curcumin/resveratrol combination in the SME formulation (CR-SME), individual curcumin in SME (C-SME), and an unformulated curcumin/resveratrol combination, in simulated gastric fluid $(n=6)$.

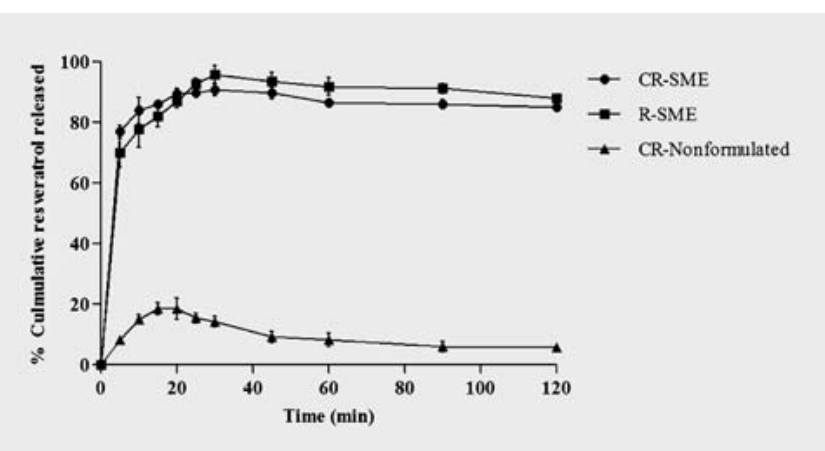

- Fig. 4 The cumulative release of resveratrol from a curcumin/ resveratrol combination in the SME formulation (CR-SME), individual resveratrol in SME (R-SME), and an unformulated curcumin/resveratrol combination, in simulated gastric fluid $(n=6)$.

CR-SME could be related to the functional groups of resveratrol. It has three hydroxyl groups and a conjugation between both the 
- Table 2 Stability data of the CR-SME in intermediate $\left(30 \pm 2^{\circ} \mathrm{C} / 65 \pm 5 \% \mathrm{RH}\right)$ and accelerated conditions $\left(45 \pm 2^{\circ} \mathrm{C} / 75 \pm 5 \% \mathrm{RH}\right)$, mean $\pm \mathrm{S}$. D. $(n=3)$.

\begin{tabular}{|c|c|c|c|c|c|c|}
\hline \multirow[t]{2}{*}{ Sampling time } & \multirow[t]{2}{*}{ Appearance } & \multirow{2}{*}{$\begin{array}{l}\text { Visual } \\
\text { grading }\end{array}$} & \multirow[t]{2}{*}{ Particle size (nm) } & \multirow[t]{2}{*}{ PDI } & \multicolumn{2}{|c|}{ \% Drug content } \\
\hline & & & & & Curcumin & Resveratrol \\
\hline - 0 month & Clear yellow liquid & A & $18.29 \pm 0.04$ & $0.02 \pm 0.01$ & $101.12 \pm 2.73$ & $99.83 \pm 4.11$ \\
\hline \multicolumn{7}{|l|}{ A) $30^{\circ} \mathrm{C} / 65 \% \mathrm{RH}$} \\
\hline - 1 month & Clear yellow liquid & A & $19.40 \pm 0.01$ & $0.04 \pm 0.02$ & $99.45 \pm 3.57$ & $102.36 \pm 3.13$ \\
\hline - 3 months & Clear yellow liquid & A & $20.22 \pm 0.03$ & $0.07 \pm 0.01$ & $101.35 \pm 4.22$ & $101.45 \pm 5.44$ \\
\hline \multicolumn{7}{|l|}{ B) $45^{\circ} \mathrm{C} / 75 \% \mathrm{RH}$} \\
\hline - 1 month & Clear yellow liquid & A & $20.45 \pm 0.05$ & $0.06 \pm 0.01$ & $99.43 \pm 3.09$ & $98.83 \pm 4.85$ \\
\hline - 3 months & Clear yellow liquid & A & $21.51 \pm 0.04$ & $0.05 \pm 0.00$ & $96.12 \pm 2.67$ & $97.72 \pm 5.14$ \\
\hline
\end{tabular}

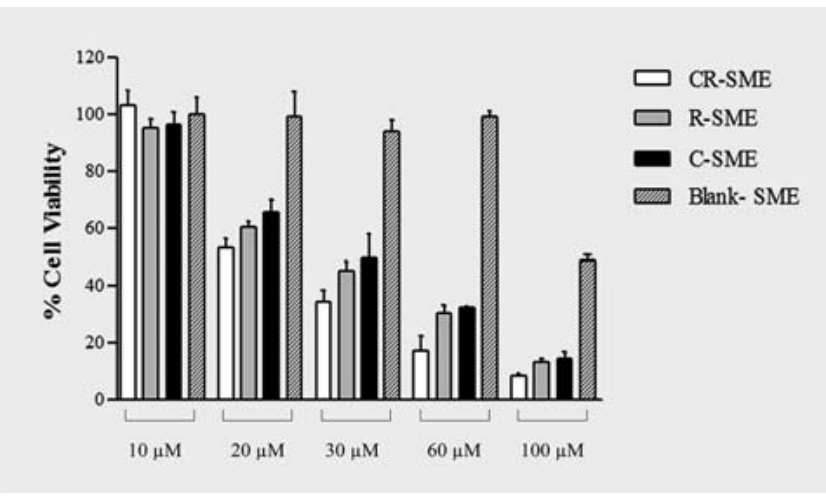

- Fig. 5 Cytotoxicity of HT-29 cells treated with different concentrations of CR-SME (each polyphenol at 5:5, 10:10, 15:15, 30:30, and $50: 50 \mu \mathrm{M}$ ) compared to the C-SME, R-SME (individual compound at $10,20,30,60$, and $100 \mu \mathrm{M})$, and blank SME; $(n=8)$, duplications.

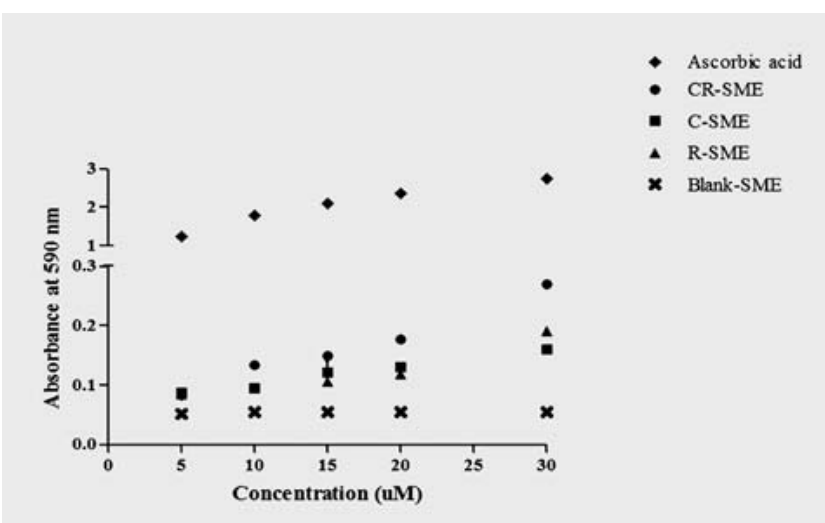

- Fig. 6 Total antioxidant power of CR-SME, C-SME, and R-SME in comparison with ascorbic acid $(5-30 \mu \mathrm{g} / \mathrm{ml})$ measured by the ferric reducing antioxidant power (FRAP) assay.

aromatic rings [15]. Similarly, the phenolic and the methoxy group on the phenyl ring and the 1,3-diketone system are important for contributing to the antioxidant activity of curcumin. When both compounds were present, there was a potential for synergy that one antioxidant helped regenerate the other. In a previous study, the decay kinetics of a curcumin/resveratrol combination was also found to be lower than each individual compound [16].

Finally, in order to evaluate whether the combination administration of curcumin and resveratrol affected the oral absorption, studies were performed on CR-SME, C-SME plus R-SME, and curcumin/resveratrol suspensions. The oral absorption of the combination or individual compound in SME was much greater than that of the combination in suspension form, as shown in $>$ Figs. 7 and 8 for curcumin and resveratrol, respectively. The plasma concentration time profiles were similar between the administration of CR-SME and C-SME following with R-SME. The pharmacokinetic parameters in rabbits are summarized in $>$ Table 3 . The total plasma concentrations of each compound from CR-SME were significantly higher when compared with the curcumin/resveratrol suspension $(\mathrm{p}<0.05)$. The $\mathrm{AUC}_{0-6 \mathrm{~h}}$ of $\mathrm{CR}-\mathrm{SME}$ increased by about 13.7-fold for resveratrol and 34.5-fold for curcumin than that of the suspension. The CR-SME administration gave a significantly higher $\mathrm{AUC}_{0-6 \mathrm{~h}}$ of curcumin than did C-SME following with R-SME $(p<0.05)$. However, there was no significant difference in the $\mathrm{AUC}_{0-6 \mathrm{~h}}$ of resveratrol between these two administration methods. In addition, all of the treatments have similar $\mathrm{T}_{\max }$ values at $60 \mathrm{~min}$ for curcumin and $90 \mathrm{~min}$ for resveratrol.

\section{Materials and Methods}

\section{Chemicals}

Curcumin (purity $\geq 70 \%$ ) was from Sigma-Aldrich. Trans-resveratrol (purity $\geq 98 \%$ ) (P. cuspidatum root extract resveratrol powder) was from Pioneer Herb. Capryol 90, Labrafac CC, Labrasol, Lauroglycol FCC, Labrafil M 2125 CS, and Lauroglycol 90 were from Gattefosse. Cremophor EL and Cremophor RH 40 were from BASF. PEG 400 and propylene glycol (PG) were from the PC Drug Center Co., Ltd. Corn oil was from the Thai Vegetable Oil Public Company limited. Trichloroacetic acid, sodium phosphate dibasic, sodium phosphate monobasic, acetonitrile, and methanol (HPLC grade) were from RCI Labscan. Potassium ferricyanide was from Ajax Finechem Pty Ltd. Ferric chloride was from Sigma-Aldrich. Ascorbic acid was from Chem-Supply Pty Ltd. Hard gelatin capsules (size 00) were from Capsugel. All other chemicals used were of analytical grade. 


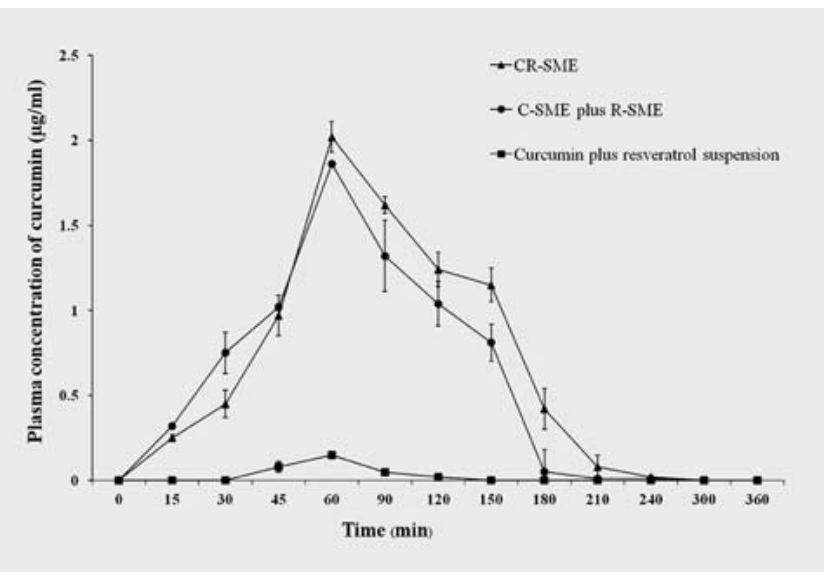

- Fig. 7 Curcumin plasma concentration vs. time profiles after oral administration of curcumin $(50 \mathrm{mg} / \mathrm{kg}$ ) in combination with resveratrol $(50 \mathrm{mg} / \mathrm{kg})$ in the SME formulation, curcumin in combination with resveratrol in aqueous suspension, or curcumin $(50 \mathrm{mg} / \mathrm{kg}$ ) SME formulation followed by a resveratrol $(50 \mathrm{mg} / \mathrm{kg}$ ) SME formulation. All values reported are mean values $\pm S D(n=3)$.

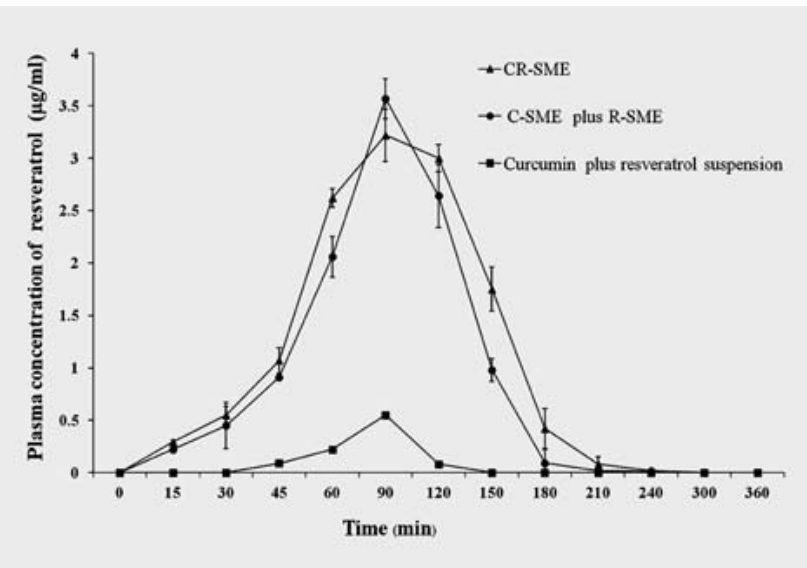

- Fig. 8 Resveratrol plasma concentration vs. time profiles after oral administration of curcumin $(50 \mathrm{mg} / \mathrm{kg})$ in combination with resveratrol $(50 \mathrm{mg} / \mathrm{kg})$ in the SME formulation, curcumin in combination with resveratrol in aqueous suspension, or a curcumin (50 mg/ kg) SME formulation followed by a resveratrol $(50 \mathrm{mg} / \mathrm{kg})$ SME formulation. All values reported are mean values \pm SD $(n=3)$.

\section{Cell culture}

Human colon adenocarcinoma cell lines (HT-29 cells; HTB-38) were from ATCC. McCoy's $5 \mathrm{a}$, FBS, and penicillin (100 IU/mL)streptomycin $(100 \mathrm{mg} / \mathrm{mL}$ ) (pen-strep) were from Gibco, Invitrogen. Trypsin-EDTA $0.25 \%$ was from Gibco, Invitrogen. MTT was from Molecular Probes, Invitrogen. PBS (pH 7.4), 2-(N-Morpholino) ethanesulfonic acid (MES) sodium salt, was from Sigma. DMSO was from Amresco.

\section{Solubility measurement}

The shake flask method was utilized to study the equilibrium solubility of the curcumin/resveratrol combinations in different oils (corn oil, Capryol 90, Labrafac PG, Labrafac CC, soyabean oil), surfactants (Capryol PGMC, Cremophor EL, Cremophor RH40, Labrafil M 2125 CS, Lauroglycol 90) and co-surfactants (Labrasol, Lauroglycol FCC, PEG 400, propylene glycol, Transcutol HP). An excess amount of curcumin and resveratrol in the ratio of $1: 1$ was added to each Eppendorf tube containing $1 \mathrm{~g}$ of the vehicle. The sample was vortexed at a maximum speed for 10 min using a mixer (Vortex-gene 2, Becthai Bangkok Equipment \& Chemical) and allowed to equilibrate in a water bath shaker (Heto Lab, Scientific Promotion) $\left(37^{\circ} \mathrm{C}\right.$ at $\left.100 \mathrm{rpm}\right)$; the equilibration time was set at $48 \mathrm{~h}$. Solid-phase separation was achieved using centrifugation $\left(30 \mathrm{~min}, 1000 \mathrm{rpm}\right.$ at $37^{\circ} \mathrm{C}$ ) and filtration $[0.2 \mu \mathrm{m}$ polyvinylidenedifluoride (PVDF) filter]. The supernatants were collected and diluted with the mobile phase [acetonitrile and $1 \%$ citric acid (v/v) (55:45)] for quantification of curcumin and resveratrol by the HPLC method. All solubility experiments were performed in triplicate.

\section{Ternary phase assay}

The compositions of oil, surfactant, and co-surfactant from the solubility study were selected to construct ternary phase diagrams. The ternary phase diagrams were plotted to identify the self-microemulsifying regions and to find the optimal concentrations of components. The mixture series of oil, surfactant, and cosurfactant were prepared. The vehicles were weighed into glass test tubes and mixed using a vortex mixer. The concentration range of each component was $10-50 \%$ oil, $25-90 \%$ surfactant, and $0-25 \%$ co-surfactant. One gram of each mixture was dispersed in $20 \mathrm{~mL}$ of distilled water. The efficiency of the self-microemulsification was observed visually and scored according to the grading system described by Singh et al. [17].

\section{Preparation of curcumin/resveratrol in self-microemulsifying formulations}

According to the ternary phase diagram studies, the SME formulation at the optimal component ratios was selected for the incorporation of curcumin and resveratrol. Thirty mg of each compound was added to $1 \mathrm{~g}$ of the self-microemulsifying mixture and the dispersion was stirred continuously until a homogenous solution was formed. The formulations were left for $48 \mathrm{~h}$ at room temperature. Hard gelatin capsules (size 00 ) were manually filled with the CR-SME formulation and stored in a tightly sealed glass bottle at room temperature until examined.

\section{Emulsion droplet size and size distribution}

One gram of each formulation was diluted with DI water, SGF, and SIF (20-fold dilution). The content was gently stirred by a magnetic stirrer for $5 \mathrm{~min}$. The droplet size and the polydispersity index of the resultant microemulsion were determined by the dynamic light scattering technique using ZetaPALS, Zeta potential, and a particle size analyzer (Brookhaven Instruments Corporation). The light scattering was performed at a fixed angle of 90 at a temperature of $25^{\circ} \mathrm{C}$. The measurement time was $1 \mathrm{~min}$, and each run comprised 10 subruns.

\section{Morphological characterization}

The morphology of microemulsion formed was observed by transmission electron microscopy (TEM; JEOL). The CR-SME formula- 
- Table 3 Pharmacokinetics value of CR-SME after oral administration compared to C-SME plus R-SME and a combined suspension (equivalent to $50 \mathrm{mg} / \mathrm{kg}$ of curcumin and resveratrol), mean \pm S. D. $(n=3)$.

\begin{tabular}{|l}
\hline Formulation \\
\hline CR-SME \\
\hline C-SME plus R-SME \\
\hline Curcumin plus resveratrol suspension \\
\hline
\end{tabular}

\begin{tabular}{|l|l|}
\hline$C_{\max }(\boldsymbol{\mu g} / \mathrm{mL})$ & \\
\hline Curcumin & Resveratrol \\
\hline $2.02 \pm 0.09$ & $3.22 \pm 0.25$ \\
\hline $1.86 \pm 0.02$ & $3.57 \pm 0.19$ \\
\hline $0.19 \pm 0.06$ & $0.55 \pm 0.01$ \\
\hline
\end{tabular}

\begin{tabular}{|l|}
\hline$T_{\max }(\mathbf{m i n})$ \\
\hline Curcumin \\
\hline 60 \\
\hline 60 \\
\hline 60 \\
\hline
\end{tabular}

\begin{tabular}{|l|l|l|}
\hline & \multicolumn{2}{|l|}{ AUC $_{\mathbf{0}-6 \mathbf{h}}(\boldsymbol{\mu g ~ h} / \mathrm{mL})$} \\
\hline Resveratrol & Curcumin & Resveratrol \\
\hline 90 & $1593.75 \pm 142.53$ & $2688.75 \pm 292.65$ \\
\hline 90 & $1304.50 \pm 118.51$ & $2246.00 \pm 284.45$ \\
\hline 90 & $46.25 \pm 5.72$ & $196.25 \pm 39.29$ \\
\hline
\end{tabular}

tion was diluted with distilled water at a ratio of $1: 75$ and mixed by gentle shaking. A drop of the sample obtained after the dilution was placed on copper grids. Any excess liquid was drawn off with filter paper. The grid surface was then air-dried at room temperature.

\section{Release of curcumin and resveratrol from the self-microemulsifying formulation}

The release profiles from the capsules filled with CR-SME containing $30 \mathrm{mg}$ of each curcumin and resveratrol, C-SME containing $30 \mathrm{mg}$ of curcumin, and R-SME containing $30 \mathrm{mg}$ of resveratrol were compared to the unformulated combination. This study was carried out using the USP 30 rotating paddle apparatus with $900 \mathrm{~mL}$ of simulated gastric fluid (SGF, pH 1.2) at $37.0 \pm 0.5^{\circ} \mathrm{C}$ and $75 \mathrm{rpm}$. The prepared formulations were subjected to the release studies for $2 \mathrm{~h}$. Samples were withdrawn and replaced with the fresh medium at 5, 10, 15, 30, 45, 60, 90, and $120 \mathrm{~min}$. The concentrations of curcumin and resveratrol were assayed by HPLC. The test was repeated six times, and the data were reported as the mean \pm SD. A plot of the cumulative \% release of curcumin and resveratrol against time was constructed to illustrate the drug release profiles.

\section{HPLC analysis of curcumin in combination with resveratrol}

The analysis of curcumin in combination with resveratrol in the drug release samples was performed on an Agilent separation module with a photodiode array detector (HP 1100, Agilent). A C18 column (VertiSep ${ }^{\mathrm{TM}}$ UPS C18 column $4.6 \times 250 \mathrm{~mm}, 5 \mu \mathrm{m}$, Ligand Scientific) was used. The mobile phase consisted of a mixture of acetonitrile and $1 \%$ citric acid $(\mathrm{v} / \mathrm{v})(55: 45)$ [18] with an isocratic solvent system. The injection volume was $20 \mu \mathrm{L}$. The flow rate of the mobile phase was $1 \mathrm{~mL} / \mathrm{min}$, and the detector wavelength was kept at $325 \mathrm{~nm}$ and 425 for curcumin and resveratrol detection, respectively.

\section{Stability studies}

The stability testing was carried out according to the $\mathrm{ICH}$ guidelines (2003) on the topic of Q1 A (R2): stability testing of the new drug substances and products. The optimized CR-SME formulation was subjected to stability studies in order to evaluate its physical and chemical stability. Samples were kept in a stability chamber (Patron AH-80) under intermediate conditions [30 \pm $2{ }^{\circ} \mathrm{C}, 65 \pm 5 \%$ relative humidity $\left.(\mathrm{RH})\right]$, and evaluated under accelerated conditions $\left(45 \pm 2^{\circ} \mathrm{C}, 75 \pm 5 \% \mathrm{RH}\right)$, with the humidity and temperature control taken at 0,1 , and, 3 months for both condi- tions. Samples were prepared for the assay in the mobile phase and injected directly onto the HPLC column three separate times $(n=3)$.

\section{Ferric reducing antioxidant power assay}

The FRAP assay was used to evaluate the antioxidant capacity in order to determine the ferric reducing activity of the polyphenol co-delivery in the SME formulation. PBS $2.5 \mathrm{~mL}$ was mixed with the same amount of potassium ferricyanide and the sample solution (CR-SME, R-SME, and C-SME), standard ascorbic acid, or blank formulation was added into the tube. After incubation for $20 \mathrm{~min}$ at $50^{\circ} \mathrm{C}, 2.5 \mathrm{~mL}$ of trichloroacetic acid were added and centrifuged for $10 \mathrm{~min}$ at $3000 \mathrm{rpm}$ to separate the layers. The supernatant was transferred for mixing with $2.5 \mathrm{~mL}$ of DI water and $0.5 \mathrm{~mL}$ of ferric chloride. Finally, the absorbance of all samples, standards, and blank were measured at $590 \mathrm{~nm}$.

\section{Cell culture studies}

The HT-29 cells were grown in McCoy's 5 A (modified) medium supplemented with $10 \% \mathrm{v} / \mathrm{v} F B S$, and $1 \% \mathrm{v} / \mathrm{v}$ pen-strep. The cells were maintained at $37 \pm 0.5^{\circ} \mathrm{C}$ in an atmosphere with $5 \% \mathrm{CO}_{2}$ and $90 \% \mathrm{RH}$, and passaged every 2 days. When the cell monolayer reached $80-100 \%$ confluency, the cells were removed from the culture flask using a $0.25 \%$ trypsin-EDTA solution. Viable cell numbers were counted prior to use by a standard hemocytometer. The cells were then used in cell cytotoxicity studies.

\section{The cytotoxicity test}

To evaluate the cytotoxicity of CR-SME at different concentrations, each polyphenol in the formulation was mixed at ratios of $5: 5,10: 10,15: 15,30: 30$, and $50: 50 \mu \mathrm{M}$ to get the final concentrations of the combination at $10,20,30,60$, and $100 \mu \mathrm{M}$, respectively. Each sample was dispersed in DI water and then diluted with the complete medium. The HT-29 cells were seeded in 96well cell culture plates at a density of $8 \times 10^{3}$ cells/well and incubated for $24 \mathrm{~h}$. After overnight incubation, the culture medium was removed, and the cells were washed with $100 \mu \mathrm{L}$ of PBS. One hundred microliters of the samples at different concentrations were added to each well. Complete medium and $1 \%$ sodium lauryl sulfate were used as the negative control and positive control, respectively. After $24 \mathrm{~h}$ treatment, the samples were removed and the cells were washed with PBS. Fifty microliters of $0.5 \mathrm{mg} / \mathrm{mL}$ MTT solution were added to each well and the cells were incubated for another $4 \mathrm{~h}$. After removing the MTT solution carefully, $100 \mu \mathrm{L}$ of DMSO were added to dissolve the formazan crystals formed by the living cells. The absorbance of the samples was 
measured by a microplate reader (DTX 880 Multimode Detector, Beckman Coulter Inc.) at a wavelength of $570 \mathrm{~nm}$. Duplications were performed. The percentage of cell viability was calculated relative to the measured absorbance of the negative control that exhibited $100 \%$ cell viability.

\section{In vivo absorption studies}

The male New Zealand white rabbits with a mean body weight of $2.5 \pm 0.2 \mathrm{~kg}$ were supplied by the Animal House, Faculty of Science, Prince of Songkla University. The animal protocol was approved under the guidelines of the Animal Care and the Committee of Prince of Songkla University (MOE 0521.11/061). The overnight fasted rabbits were divided into three groups with three rabbits per each group. The compounds 1) CR-SME formulation (curcumin $50 \mathrm{mg} / \mathrm{kg}$ and resveratrol $50 \mathrm{mg} / \mathrm{kg}$ ) 2) curcumin/resveratrol in aqueous suspension (curcumin $50 \mathrm{mg} / \mathrm{kg}$ and resveratrol $50 \mathrm{mg} / \mathrm{kg}$ ), or 3) C-SME formulation $(50 \mathrm{mg} / \mathrm{kg}$ ) followed by R-SME formulation $(50 \mathrm{mg} / \mathrm{kg}$ ) were orally administered as a single dose. Blood samples $(1 \mathrm{~mL})$ were collected via the auricular artery [19] $0,15,30,45,60,90,120,180,210,240,300$, and 360 min after oral administration and were immediately transferred to a heparinized microcentrifuge tube and centrifuged at $4000 \mathrm{~g}$ for $20 \mathrm{~min}$ at $4^{\circ} \mathrm{C}$. The plasma samples were separated. Acetonitrile was added to each plasma sample (acetonitrile:plasma was $1: 1 \mathrm{v} / \mathrm{v}$ ), vortexed, sonicated, and allowed to stand for $5 \mathrm{~min}$ for deproteinization. The protein precipitate was removed by centrifugation. The supernatant was pipetted into a tube. Samples were then diluted with methanol in a ratio of $1: 0.5$. The solution was filtered using a $0.2-\mu \mathrm{m}$ membrane filter and subjected to the validated HPLC method. The pharmacokinetic parameters including the maximum concentration $\left(C_{\max }\right)$, time to reach maximum concentration $\left(T_{\max }\right)$, and the area under the concentration-time curve ( $\mathrm{AUC}_{0-6 \mathrm{~h}}$ ) were determined.

\section{Statistical analysis}

All results are expressed as the mean \pm SD. Differences between two related parameters were assessed by Student's t-test or oneway ANOVA. Differences were considered significant at $p<0.05$.

\section{Acknowledgements}

This work was supported by the National Research University Project of Thailand, Prince of Songkla University, Thailand Research Fund under the Royal Golden Jubilee Ph.D. programme (PHD/ 0262/2552), and the Excellent Research Laboratory of Cancer Molecular Biology, Prince of Songkla University. We also thank Dr. Brian Hodgson for assistance with the English.

\section{Conflict of Interest}

There is no conflict of interest.

\section{References}

[1] Bayet-Robert M, Kwiatkowski F, Leheurteur M, Gachon F, Planchat E, Abrial C, Mouret-Reynier MA, Durando X, Barthomeuf C, Chollet P. Phase
I dose escalation trial of docetaxel plus curcumin in patients with advanced and metastatic breast cancer. Cancer Biol Ther 2010; 9: 8-14

[2] Chen Y, Tseng SH, Lai HS, Chen WJ. Resveratrol-induced cellular apoptosis and cell cycle arrest in neuroblastoma cells and antitumor effects on neuroblastoma in mice. Surgery 2004; 136: 57-66

[3] Majumdar AP, Banerjee S, Nautiyal J, Patel BB, Patel V, Du J, Yu Y, Elliott AA, Levi E, Sarkar FH. Curcumin synergizes with resveratrol to inhibit coIon cancer. Nutr Cancer 2009; 61: 544-553

[4] Singh SK, Verma PR, Razdan B. Glibenclamide-loaded self-nanoemulsifying drug delivery system: development and characterization. Drug Dev Ind Pharm 2010; 36: 933-945

[5] Pathak A, Jain V, Nagariya AK, Singh R, Nayak S, Bansal P, Gupta V, Kumar $\mathrm{S}$, Singh $\mathrm{H}$. Recent advances in self emulsifying drug delivery system - A review. Drug Inv Today 2010; 2: 123-129

[6] Rajesh BV, Reddy TK, Srikanth G, Mallikarjun V, Nivethithai P. Lipid based self-emulsifying drug delivery system (SEDDS) for poorly water-soluble drugs: A review. J Global Pharma Tech 2009; 2: 47-55

[7] Setthacheewakul S, Mahattanadul S, Phadoongsombut N, Pichayakorn W, Wiwattanapatapee R. Development and evaluation of self-microemulsifying liquid and pellet formulations of curcumin, and absorption studies in rats. Eur J Pharm Biopharm 2010; 76: 475-485

[8] Haukvik T, Bruzell E, Kristensen S, Tønnesen HH. Photokilling of bacteria by curcumin in selected polyethylene glycol 400 (PEG 400) preparations studies on curcumin and curcuminoids, XLI. Pharmazie 2010; 65: 600606

[9] Gaumet M, Gurny R, Delie F. Localization and quantification of biodegradable particles in an intestinal cell model: the influence of particle size. Eur J Pharm Sci 2009; 36: 465-473

[10] Li L, Braiteh FS, Kurzrock R. Liposome-encapsulated curcumin. In vitro and in vivo effects on proliferation, apoptosis, signaling and angiogenesis. Cancer 2005; 106: 1322-1330

[11] Queimada AJ, Mota FL, Pinho SP, Macedo EA. Solubilities of biologically active phenolic compounds: measurements and modeling. J Phys Chem B 2009; 113: 3469-3476

[12] Lestari ML, Indrayanto G. Curcumin. Profiles Drug Subst Excip Relat Methodol 2014; 39: 113-204

[13] Yamaguchi JY, Nishimura Y, Kanada A, Kobayashi M, Mishima K, Tatsuishi T, Iwase K, Oyama Y. Cremophor EL, a non-ionic surfactant, promotes $\mathrm{Ca}(2+)$-dependent process of cell death in rat thymocytes. Toxicology 2005; 211: 179-186

[14] Rice-Evans CA, Miller NJ, Paganga G. Structure-antioxidant activity relationships of flavonoids and phenolic acids. Free Radic Biol Med 1996; 20 : 933-956

[15] Aggarwal BB, Bhatt ID, Ichikawa H, Ahn KS, Sethi G, Sandur SK, Sundaram C, Seeram N, Shishodia S. Curcumin - biological and medicinal properties. In: Ravindran PN, Nirmal Babu K, Sivaraman K, editors. Turmeric: The genus Curcuma. Boca Raton, FL: CRC Press; 2007: 297368

[16] Smoliga JM, Blanchard O. Enhancing the delivery of resveratrol in humans: if low bioavailability is the problem, what is the solution? Molecules 2014; 19: 17154-17172

[17] Singh AK, Chaurasiy A, Awasthi A, Mishra G, Asati D, Khar RK, Mukherjee $R$. Oral bioavailability enhancement of exemestane from self-microemulsifying drug delivery system (SMEDDS). AAPS PharmSciTech 2009; 10: 906-916

[18] Narayanan KN, Nargi D, Randolph C, Narayanan BA. Liposome encapsulation of curcumin and resveratrol in combination reduces prostate cancer incidence in PTEN knockout mice. Int J Cancer 2009; 125: 1-8

[19] Stagni G, Shukla C. Pharmacokinetics of methotrexate in rabbit skin and plasma after iv-bolus and iontophoretic administrations. J Control Release 2003; 93: 283-292 